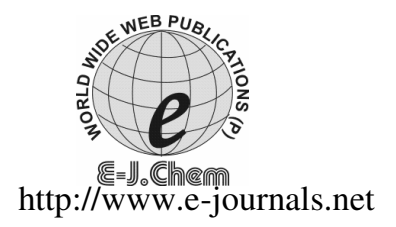

ISSN: 0973-4945; CODEN ECJHAO

E-Journal of Chemistry

2011, 8(3), 1158-1163

\title{
Synthesis and Characterization of Some Group 12 Metal Complexes with Tellurium Containing 10-Membered Tetraazamacrocyclic Ligands
}

\author{
NITU and K. K. VERMA* \\ Department of Chemistry \\ Maharshi Dayanand University, Rohtak -124001, India \\ vermakk123@rediffmail.com
}

Received 12 October 2010; Accepted 17 December 2010

\begin{abstract}
A new series of 10-membered tellurium containing tetraazamacrocyclic complexes, $\left[\mathrm{ML}^{1} \mathrm{Cl}_{2}\right],\left[\mathrm{ML}^{2} \mathrm{Cl}_{2}\right]$ and $\left[\mathrm{ML}^{3} \mathrm{Cl}_{2}\right]$, where $[\mathrm{M}=\mathrm{Zn}(\mathrm{II}), \mathrm{Cd}(\mathrm{II})$, $\mathrm{Hg}(\mathrm{II}) ; \mathrm{L}^{1}, \mathrm{~L}^{2}$ and $\mathrm{L}^{3}=10$-membered tellurium containing tetraazamacrocyclic ligands] have been prepared via the template condensation of ethylenediamine and diaryltellurium dichlorides, $\mathrm{R}_{2} \mathrm{TeCl}_{2},(\mathrm{R}=p$-hydroxyphenyl, 3-methyl-4hydroxyphenyl, $p$-methoxyphenyl) in the presence of metal chlorides. These complexes have been characterized by elemental analyses, conductivity measurements, infrared, electronic absorption and proton magnetic resonance spectral studies. IR and PMR spectra confirm the formation of 10-membered tetraazamacrocycle skeleton and their tetradentate nature. An octahedral geometry has been assigned to all the metal complexes on the basis of various physicochemical studies.
\end{abstract}

Keywords: Tellurium, Tetraazamacrocycles, Diaryltellurium dichlorides, Ethylenediamine, Template condensation.

\section{Introduction}

The coordination chemistry of organotellurium ligands containing hard donor atoms such as nitrogen and oxygen along with soft tellurium is interesting as such ligand framework can provide 'insight' into competitive coordination behaviour between the hard and soft donors towards a metal center ${ }^{1,2}$. Such molecular systems may be important in transition metal catalyzed asymmetric synthesis ${ }^{3,4}$ and as single source precursors in MOCVD processes ${ }^{5-7}$. Also, macrocycle complexes are significant as they have been found to act as possible models for biochemically important proteins and enzymes ${ }^{8-12}$.

Some recent publications ${ }^{13-17}$ exhibit the development of tellurium containing macrocycles. Srivastava et al. ${ }^{18}$ reported the route of synthesis of metal complexes with tellurium containing 
macrocycles. In continuation of our earlier work ${ }^{19,20}$ on such macrocyclic complexes, we herein report the synthesis and characterization of divalent zinc, cadmium and mercury complexes with three novel tellurium tetraazamacrocycles, $\left(\mathrm{Te}_{2} \mathrm{~N}_{4}\right.$ system).

\section{Experimental}

The chemicals, tellurium tetrachloride (Aldrich), ethylenediamine, $\mathrm{ZnCl}_{2}, \mathrm{CdCl}_{2} .2 .5 \mathrm{H}_{2} \mathrm{O}$, $\mathrm{HgCl}_{2}$, phenol, $o$-cresol and anisole (Loba Chemie) were of reagent grade. All the solvents were purified and dried before use by conventional methods.

\section{Preparation of diaryltellurium dichlorides}

Bis( $p$-hydroxyphenyl)-, bis(3-methyl-4-hydroxyphenyl) and bis( $p$-methoxyphenyl) tellurium dichlorides were prepared by direct reactions of $\mathrm{TeCl}_{4}$ with phenol ${ }^{21}, o$-cresol ${ }^{22}$ and anisole ${ }^{23}$ respectively as reported in the literature. All the preparations were carried out in dry conditions under an atmosphere of nitrogen.

\section{Preparation of complexes}

The 10-membered tellurium tetraazamacrocyclic metal complexes have been synthesized by the template condensation reaction of metal chlorides with diaryltellurium dichlorides and ethylenediamine in 1:2: 2 molar ratios.

A general method for the preparation of these 1, 6-diaryltellura -2, 5, 7, 10-tetraazacyclodecane metal complexes is given below: A saturated solution of $4.0 \mathrm{mmol}$ of diaryltellurium dichloride in methanol was added slowly and with constant stirring to a methanolic $(\sim 5 \mathrm{~mL})$ solution of $4.0 \mathrm{mmol}$ of ethylenediamine taken in a R.B. flask. A distinct change in colour with slight turbidity was observed. The contents were refluxed for 2-3 $\mathrm{h}$ followed by addition of a saturated methanolic solution of $2.0 \mathrm{mmol}$ of metal dichloride. An immediate change in colour was observed. The solution was then refluxed for 3-4 $\mathrm{h}$ and concentrated to about one third of the original volume and cooled. This resulted in the separation of a coloured solid. This was filtered, washed with benzene/petroleum ether and dried in a vacuum desiccator over $\mathrm{P}_{4} \mathrm{O}_{10}$.

\section{Physical measurements}

Elemental analysis for $\mathrm{C}, \mathrm{H}, \mathrm{N}$ for the zinc and cadmium complexes were obtained from SAIF, Panjab University, Chandigarh on a CARLO ERBA Model 1106. Tellurium and chlorine contents were determined volumetrically ${ }^{24}$. Metals were estimated on atomic absorption spectrophotometer (ECIL Model No. 4129). The conductance measurements in DMSO were performed on a systronic type 305 conductivity bridge at $25 \pm 2{ }^{\circ} \mathrm{C}$ using a dip type cell with smooth platinum electrodes. The electronic spectra were recorded in dimethylsulphoxide at $25 \pm 2{ }^{\circ} \mathrm{C}$ on a thermo spectronic UV-1 spectrophotometer. The IR spectra (4000-400 $\mathrm{cm}^{-1}$ ) were recorded as $\mathrm{KBr}$ pellets on a Perkin Elmer Model 2000 FTIR Spectrometer at SAIF, Panjab University, Chandigarh. ${ }^{1} \mathrm{H}$ NMR spectra were recorded in DMSO $-\mathrm{d}_{6}$ on BRUKER XWIN - NMR AVANCE 300 operating at $300.13 \mathrm{MHz}$, using tetramethylsilane as an internal reference. The NMR spectra were obtained from Kurukshetra University, Kurukshetra.

\section{Results and Discussion}

Diaryltellurium dichlorides when condensed with ethylenediamine in presence of metal dichlorides in 2: 2: 1 molar ratios, yield the desired complexes as shown below 


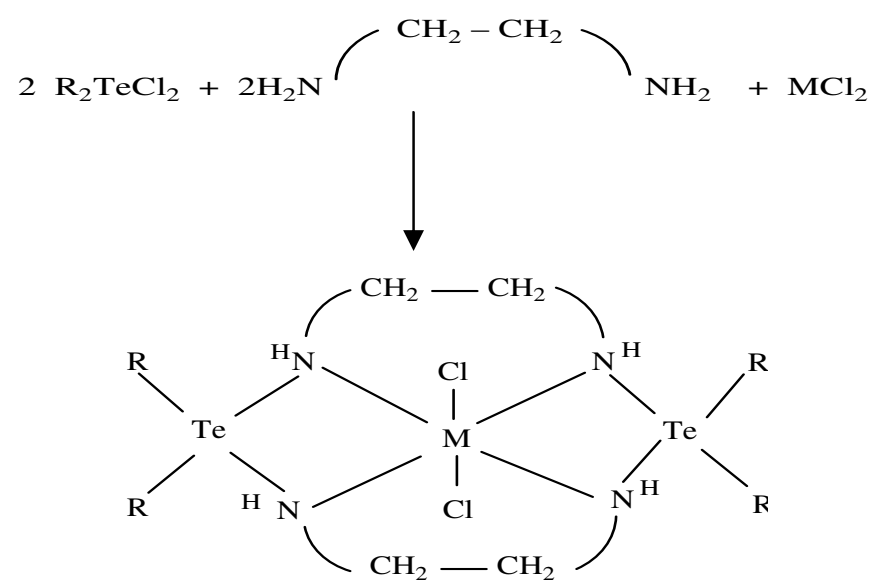

Where

$\mathrm{L}^{1} \quad ; \mathrm{R}=p$-hydroxyphyenyl

$\mathrm{L}^{2} \quad ; \mathrm{R}=3$-methyl-4-hydroxyphyenyl

$\mathrm{L}^{3} \quad ; \mathrm{R}=p$-methoxyphenyl

$\mathrm{M} \quad=\mathrm{Zn}^{\mathrm{II}}, \mathrm{Cd}^{\mathrm{II}}$ and $\mathrm{Hg}^{\mathrm{II}}$

These complexes are coloured, crystalline, air stable solids and are soluble only in polar donor organic solvents. The analytical data and some physical properties of these complexes are given in Table 1 .

\section{Conductance studies}

The molar conductance values for these complexes (except $\mathrm{ZnL}^{2} \mathrm{Cl}_{2}$ and $\mathrm{HgL}^{2} \mathrm{Cl}_{2}$ ) in DMSO at $c a .10^{-3} \mathrm{M}$ are lower than the ranges reported ${ }^{25}$ for $1: 1$ type electrolytes, reflecting their partial dissociation in this solvent. The molar conductance for $\mathrm{ZnL}^{2} \mathrm{Cl}_{2}$ is close to those of 1:1 electrolytes and for $\mathrm{HgL}^{2} \mathrm{Cl}_{2}$ is higher than those of $1: 1$ type electrolytes. These two complexes probably ionize into $\left[\mathrm{ML}^{2} \mathrm{Cl} . \mathrm{DMSO}\right]^{+}$and $\mathrm{Cl}^{-}$ions due to the solvation process, since DMSO is known to be a good donor ${ }^{25}$. So, on the basis of conductance studies, these complexes in general may be represented as $\left[\mathrm{MLCl}_{2}\right]$.

\section{Magnetic and electronic spectral studies}

As expected, these $\mathrm{d}^{10}$ metal ion complexes are diamagnetic in nature. No $\mathrm{d}-\mathrm{d}$ transitions are observed for these complexes consistent with the $\mathrm{d}^{10}$ configuration. The electronic absorption spectra of these complexes display a broad and intense band in the region $32000-22000 \mathrm{~cm}^{-1}$ due to CT transitions ${ }^{26,27}$. The $\mathrm{n}-\pi^{*}$ and $\pi-\pi^{*}$ bands of the ligand may also be mixed with this charge transfer band ${ }^{27}$. This band sweeps the near UV portion of electromagnetic radiation and extends in the visible region, with successively diminishing absorbance up to $500 \mathrm{~nm}$. This is responsible for the colour of the complexes.

\section{Infra-red spectra}

The important IR bands and their assignments are tabulated in Table 2. The spectra are quite complex and an attempt has thus been made to draw the conclusions by comparing the spectra of metal complexes with those of corresponding constituent diarytellurium dichlorides and ethylenediamine. 
Table 1. Analytical data, physical properties, yields and molar conductance for metal complexes

\begin{tabular}{|c|c|c|c|c|c|c|c|c|c|c|c|}
\hline \multirow{2}{*}{ Complex } & \multirow{2}{*}{$\begin{array}{l}\text { Empirical Formula } \\
\text { (Formula Weight) }\end{array}$} & \multirow[b]{2}{*}{ Colour } & \multirow{2}{*}{\multicolumn{2}{|c|}{$\begin{array}{c}\text { M.P. }{ }^{\circ} \mathrm{C} \\
\text { (dec.) }\end{array}$}} & \multicolumn{6}{|c|}{ Analysis found (calculated), $\%$} & \multirow{2}{*}{$\begin{array}{c}\Lambda_{\mathrm{M}} \text { at } c a \cdot 10^{-3} \mathrm{M} \mathrm{ohm}^{-1} \\
\mathrm{~cm}^{2} \mathrm{~mol}^{-1} \text { in } \mathrm{MSO}\end{array}$} \\
\hline & & & & & $\mathrm{C}$ & $\mathrm{H}$ & $\mathrm{N}$ & $\mathrm{Cl}$ & $\mathrm{Te}$ & $\mathrm{M}$ & \\
\hline$\left[\mathrm{ZnL}^{1} \mathrm{Cl}_{2}\right]$ & $\begin{array}{c}\mathrm{C}_{28} \mathrm{H}_{32} \mathrm{Cl}_{2} \mathrm{~N}_{4} \\
\mathrm{O}_{4} \mathrm{Te}_{2} \mathrm{Zn}(880.1)\end{array}$ & $\begin{array}{l}\text { Bright } \\
\text { orange }\end{array}$ & 100-101 & 82 & $\begin{array}{c}38.01 \\
(38.21)\end{array}$ & $\begin{array}{c}3.16 \\
(3.66)\end{array}$ & $\begin{array}{c}6.13 \\
(6.37)\end{array}$ & $\begin{array}{c}8.44 \\
(8.06)\end{array}$ & $\begin{array}{l}28.93 \\
(29.00)\end{array}$ & $\begin{array}{l}7.36 \\
(7.43)\end{array}$ & 26.7 \\
\hline$\left[\mathrm{ZnL}^{2} \mathrm{Cl}_{2}\right]$ & $\begin{array}{c}\mathrm{C}_{32} \mathrm{H}_{40} \mathrm{Cl}_{2} \mathrm{~N}_{4} \\
\mathrm{O}_{4} \mathrm{Te}_{2} \mathrm{Zn}(936.2)\end{array}$ & Brown & $158-160$ & 82 & $\begin{array}{l}40.80 \\
(41.05)\end{array}$ & $\begin{array}{c}4.14 \\
(4.31)\end{array}$ & $\begin{array}{c}5.62 \\
(5.98)\end{array}$ & $\begin{array}{c}7.62 \\
(7.57)\end{array}$ & $\begin{array}{l}27.65 \\
(27.26)\end{array}$ & $\begin{array}{c}6.91 \\
(6.98)\end{array}$ & 63.4 \\
\hline$\left[\mathrm{ZnL}^{3} \mathrm{Cl}_{2}\right]$ & $\begin{array}{c}\mathrm{C}_{32} \mathrm{H}_{40} \mathrm{Cl}_{2} \mathrm{~N}_{4} \\
\mathrm{O}_{4} \mathrm{Te}_{2} \mathrm{Zn}(936.2)\end{array}$ & $\begin{array}{l}\text { Pale } \\
\text { white }\end{array}$ & $118-120$ & 63 & $\begin{array}{c}40.91 \\
(41.05)\end{array}$ & $\begin{array}{c}4.21 \\
(4.31)\end{array}$ & $\begin{array}{c}5.76 \\
(5.98)\end{array}$ & $\begin{array}{c}7.49 \\
(7.57)\end{array}$ & $\begin{array}{l}27.35 \\
(27.26)\end{array}$ & $\begin{array}{l}6.85 \\
(6.98)\end{array}$ & 20.2 \\
\hline$\left[\mathrm{CdL}^{1} \mathrm{Cl}_{2}\right]$ & $\begin{array}{c}\mathrm{C}_{28} \mathrm{H}_{32} \mathrm{Cl}_{2} \mathrm{~N}_{4} \\
\mathrm{O}_{4} \mathrm{Te}_{2} \mathrm{Cd}(927.1)\end{array}$ & $\begin{array}{l}\text { Dark } \\
\text { red }\end{array}$ & $178-180$ & 48 & $\begin{array}{l}36.03 \\
(36.27)\end{array}$ & $\begin{array}{l}3.08 \\
(3.48)\end{array}$ & $\begin{array}{c}5.82 \\
(6.94)\end{array}$ & $\begin{array}{c}7.50 \\
(7.65)\end{array}$ & $\begin{array}{l}27.65 \\
(27.53)\end{array}$ & $\begin{array}{l}11.55 \\
(12.13)\end{array}$ & 37.5 \\
\hline$\left[\mathrm{CdL}^{2} \mathrm{Cl}_{2}\right]$ & $\begin{array}{c}\mathrm{C}_{32} \mathrm{H}_{40} \mathrm{Cl}_{2} \mathrm{~N}_{4} \\
\mathrm{O}_{4} \mathrm{Te}_{2} \mathrm{Cd}(983.2)\end{array}$ & $\begin{array}{l}\text { Orange } \\
\text { pink }\end{array}$ & $180-185$ & 77 & $\begin{array}{c}38.62 \\
(39.09)\end{array}$ & $\begin{array}{c}3.89 \\
(4.10)\end{array}$ & $\begin{array}{c}5.16 \\
(5.70)\end{array}$ & $\begin{array}{l}7.39 \\
(7.21)\end{array}$ & $\begin{array}{l}25.50 \\
(25.96)\end{array}$ & $\begin{array}{c}10.92 \\
(11.43)\end{array}$ & 33.6 \\
\hline$\left[\mathrm{CdL}^{3} \mathrm{Cl}_{2}\right]$ & $\begin{array}{c}\mathrm{C}_{32} \mathrm{H}_{40} \mathrm{Cl}_{2} \mathrm{~N}_{4} \\
\mathrm{O}_{4} \mathrm{Te}_{2} \mathrm{Cd}(983.2)\end{array}$ & $\begin{array}{l}\text { Light } \\
\text { brown }\end{array}$ & $117-121$ & 65 & $\begin{array}{c}38.91 \\
(39.09)\end{array}$ & $\begin{array}{c}3.97 \\
(4.10)\end{array}$ & $\begin{array}{c}5.58 \\
(5.70)\end{array}$ & $\begin{array}{c}7.02 \\
(7.21)\end{array}$ & $\begin{array}{l}25.84 \\
(25.96)\end{array}$ & $\begin{array}{l}11.31 \\
(11.43)\end{array}$ & 22.5 \\
\hline$\left[\mathrm{HgLL}^{1} \mathrm{Cl}_{2}\right]$ & $\begin{array}{c}\mathrm{C}_{28} \mathrm{H}_{32} \mathrm{Cl}_{2} \mathrm{~N}_{4} \\
\mathrm{O}_{4} \mathrm{Te}_{2} \mathrm{Hg}(1015.3)\end{array}$ & Red & $130-133$ & 52 & (33.12) & $(3.18)$ & $(5.52)$ & $\begin{array}{c}6.80 \\
(6.98)\end{array}$ & $\begin{array}{l}25.14 \\
(25.55)\end{array}$ & $\begin{array}{l}19.69 \\
(19.76)\end{array}$ & 60.7 \\
\hline$\left[\mathrm{HgL}^{2} \mathrm{Cl}_{2}\right]$ & $\begin{array}{c}\mathrm{C}_{32} \mathrm{H}_{40} \mathrm{Cl}_{2} \mathrm{~N}_{4} \\
\mathrm{O}_{4} \mathrm{Te}_{2} \mathrm{Hg}(1071.4)\end{array}$ & $\begin{array}{c}\text { Dark } \\
\text { brown }\end{array}$ & $120-125$ & 67 & $(35.87)$ & $(3.76)$ & (5.23) & $\begin{array}{c}6.50 \\
(6.62)\end{array}$ & $\begin{array}{c}23.72 \\
(23.82)\end{array}$ & $\begin{array}{c}18.26 \\
(18.72)\end{array}$ & 108.4 \\
\hline$\left[\mathrm{HgL}^{3} \mathrm{Cl}_{2}\right]$ & $\begin{array}{c}\mathrm{C}_{32} \mathrm{H}_{40} \mathrm{Cl}_{2} \mathrm{~N}_{4} \\
\mathrm{O}_{4} \mathrm{Te}_{2} \mathrm{Hg}(1071.4)\end{array}$ & Brown & $108-110$ & 51 & (35.87) & (3.76) & (5.23) & $\begin{array}{c}6.53 \\
(6.62) \\
\end{array}$ & $\begin{array}{l}23.75 \\
(23.82) \\
\end{array}$ & $\begin{array}{c}18.55 \\
(18.72) \\
\end{array}$ & 46.5 \\
\hline
\end{tabular}

Table 2. Important IR data $\left(\mathrm{cm}^{-1}\right)$ for metal complexes

\begin{tabular}{ccccc}
\hline Complex & $v_{\mathrm{N}-\mathrm{H}}$ & N-H def. & N-H out of the plane bending & $\mathrm{v}_{\mathrm{C}-\mathrm{N}}$ \\
\hline$\left[\mathrm{ZnL}^{1} \mathrm{Cl}_{2}\right]$ & $3145 \mathrm{~m}$ & $1625 \mathrm{sh}$ & $825 \mathrm{~s}$ & $1174 \mathrm{~s}$ \\
{$\left[\mathrm{ZnL}^{2} \mathrm{Cl}_{2}\right]$} & $3160 \mathrm{~m}$ & $1640 \mathrm{~m}$ & $813 \mathrm{~s}$ & $1174 \mathrm{~m}$ \\
{$\left[\mathrm{ZnL}^{3} \mathrm{Cl}_{2}\right]$} & $3240 \mathrm{~m}$ & $1651 \mathrm{~m}$ & $822 \mathrm{~s}$ & $1176 \mathrm{~s}$ \\
{$\left[\mathrm{CdL}^{1} \mathrm{Cl}_{2}\right]$} & $3155 \mathrm{~m}$ & $1631 \mathrm{~m}$ & $828 \mathrm{~s}$ & $1172 \mathrm{~s}$ \\
{$\left[\mathrm{CdL}^{2} \mathrm{Cl}_{2}\right]$} & $3190 \mathrm{~m}$ & $1635 \mathrm{~m}$ & $813 \mathrm{~s}$ & $1174 \mathrm{~m}$ \\
{$\left[\mathrm{CdL}^{3} \mathrm{Cl}_{2}\right]$} & $3260 \mathrm{~m}$ & $1652 \mathrm{~m}$ & $822 \mathrm{~s}$ & $1176 \mathrm{vs}$ \\
{$\left[\mathrm{HgL}^{1} \mathrm{Cl}_{2}\right]$} & $*$ & $1635 \mathrm{~m}$ & $825 \mathrm{vs}$ & $1172 \mathrm{~s}$ \\
{$\left[\mathrm{HgL}^{2} \mathrm{Cl}_{2}\right]$} & $3165 \mathrm{~m}$ & $1640 \mathrm{sh}$ & $813 \mathrm{~m}$ & $1175 \mathrm{~m}$ \\
{$\left[\mathrm{HgL}^{3} \mathrm{Cl}_{2}\right]$} & $3250 \mathrm{~m}$ & $1654 \mathrm{~m}$ & $821 \mathrm{~s}$ & $1176 \mathrm{~s}$ \\
\hline
\end{tabular}

*mixed with $v_{O-H}, s=$ strong, $m=$ medium, vs. = very strong, $s h=$ shoulder 
The metal complexes under study did not show bands characteristics of free $\mathrm{NH}_{2}$ group, instead the entire complexes exhibit a single sharp absorption band at around $3145-3260 \mathrm{~cm}^{-1}$ (sometimes mixed with $\mathrm{O}-\mathrm{H}$ ) attributed to $\mathrm{v}_{\mathrm{N}-\mathrm{H}}$ stretching vibration. The assignment of this sharp band is based on the fact that macrocyclic ligands which have coordinated secondary amino group, have bands ${ }^{18,28-30}$ in the vicinity of $3200 \mathrm{~cm}^{-1}$. This contention finds support ${ }^{28}$ from appearance of bands of medium to strong intensity at $\sim 1640 \mathrm{~cm}^{-1}$ and $\sim 825 \mathrm{~cm}^{-1}$ assigned as N-H deformation coupled with N-H out of the plane bending vibrations. Bands at $\sim 1175 \mathrm{~cm}^{-1}$ may be reasonably assigned to $\mathrm{C}-\mathrm{N}$ stretching vibration ${ }^{18,31,32}$. The above observation strongly suggest ${ }^{18,28,31,32}$ that proposed macrocyclic framework is formed. The formation of tellurium containing macrocyclic ring is also supported by appearance of new weak intensity bands around $420-410 \mathrm{~cm}^{-1}$ due to Te- $\mathrm{N}^{18,33}$. Evidence for formation of proposed macrocycles and coordination through $\mathrm{N}$ atoms is further supported by new medium to weak intensity bands around $480-450 \mathrm{~cm}^{-1}$ assignable to $\mathrm{M}-\mathrm{N}$ stretching ${ }^{34}$. The $\mathrm{M}-\mathrm{Cl}, \mathrm{Cd}-\mathrm{N}$ and $\mathrm{Hg}-\mathrm{N}$ vibrations could not be ascertained due to non-availability of far infrared data.

\section{Proton magnetic resonance spectra}

The proton chemical shifts for some representative complexes which are soluble in DMSO- $\mathrm{d}_{6}$ are compiled in Table 3. The phenyl protons in metal complexes resonate at slightly up field side $(6.92-7.65 \delta \mathrm{ppm})$ as compared to parent diaryltellurium dichlorides ${ }^{21,22,35}$, due to increase in electron density at the tellurium atom as a result of replacement of $2 \mathrm{Cl}$ by $2 \mathrm{~N}$ atoms. Ethylenediamine, $\stackrel{\text { (a) }}{\mathrm{H}_{2}} \mathrm{~N}-\left(\stackrel{\text { (b) }}{\mathrm{CH}_{2}}\right)-\stackrel{\text { (a) }}{\mathrm{NH}_{2}}$ shows ${ }^{36}$ two sets of four equivalent protons each at $(\mathrm{a})=1.19 \delta \mathrm{ppm}$ and $(\mathrm{b})=2.74 \delta \mathrm{ppm}$. Metal complexes do not show any signal attributable to free $-\mathrm{NH}_{2}$, instead a broad singlet at around 1.50-2.05 $\delta \mathrm{ppm}$, which may be assigned to coordinated secondary amino group ${ }^{37}$ is observed. This confirms the formation of proposed macrocycle skeleton. The deshielding of $-\mathrm{NH}-$ protons further suggests the donation of electron density to the metal-ions. The methylene protons in these metal complexes resonate at $2.05-2.50 \delta \mathrm{ppm}$, as a multiplet as reported ${ }^{18,30,32}$ for other tetraazamacrocyles derived from ethylenediamine. Also, the independence of aryl proton chemical shifts on the nature of metal-ion precludes the possibility of Te-M bond. Thus, PMR studies on these metal complexes support the formation of 10-membered tellurium tetraazamacrocycles and their tetra dentate ligation behaviour as predicted by IR studies.

Table 3. PMR data $(\delta \mathrm{ppm})$ for metal complexes in DMSO- $\mathrm{d}_{6}$

\begin{tabular}{|c|c|c|c|c|c|c|}
\hline Complex & $-\mathrm{NH}-$ & $-\mathrm{CH}_{3}^{*}$ & $-\mathrm{OCH}_{3}$ & $-\mathrm{CH}_{2-}$ & Phenyl & $-\mathrm{OH}$ \\
\hline$\left[\mathrm{ZnL}^{2} \mathrm{Cl}_{2}\right]$ & $2.05^{\mathrm{s}}$ & $2.42^{\mathrm{s}}(12 \mathrm{H})$ & --- & $2.14^{\mathrm{m}}(8 \mathrm{H})$ & $\begin{array}{c}6.92^{\mathrm{d}}(4 \mathrm{H}) \\
7.59^{\mathrm{d}}, 7.56^{\mathrm{s}}(8 \mathrm{H})\end{array}$ & $9.95^{\mathrm{b}}(4 \mathrm{H})$ \\
\hline$\left[\mathrm{CdL}^{2} \mathrm{Cl}_{2}\right]$ & $1.50^{\mathrm{s}}($ & $2.49^{\mathrm{s}}(12 \mathrm{H})$ & --- & $2.05^{\mathrm{m}}(8 \mathrm{H})$ & $\begin{array}{c}6.94^{\mathrm{d}}(4 \mathrm{H}) \\
7.47^{\mathrm{s}}, 7.56^{\mathrm{d}}(8 \mathrm{H})\end{array}$ & $8.17^{\mathrm{b}}(4 \mathrm{H})$ \\
\hline$\left[\mathrm{HgL}^{2} \mathrm{Cl}_{2}\right]$ & $1.60^{\mathrm{s}}(4 \mathrm{H})$ & $2.50^{\mathrm{s}}(12 \mathrm{H})$ & --- & $2.12^{\mathrm{m}}(8 \mathrm{H})$ & $\begin{array}{c}6.92^{\mathrm{d}}(4 \mathrm{H}) \\
7.56^{\mathrm{d}}, 7.65^{\mathrm{s}}(8 \mathrm{H})\end{array}$ & $10.19^{\mathrm{b}}(4 \mathrm{H})$ \\
\hline$\left[\mathrm{ZnL}^{3} \mathrm{Cl}_{2}\right.$ & $1.79^{\mathrm{s}}(4 \mathrm{H})$ & --- & $3.79^{\mathrm{s}}(12 \mathrm{H})$ & $2.50^{\mathrm{m}}(8 \mathrm{H})$ & $7.16^{\mathrm{d}}(8 \mathrm{H}) 7.41^{\mathrm{d}}(8 \mathrm{H})$ & --- \\
\hline$\left[\mathrm{CdL}^{3} \mathrm{Cl}_{2}\right]$ & $1.75^{\mathrm{s}}(4 \mathrm{H})$ & --- & $3.78^{\mathrm{s}}(12 \mathrm{H})$ & $2.50^{\mathrm{m}}(8 \mathrm{H})$ & $7.16^{\mathrm{d}}(8 \mathrm{H}) 7.74^{\mathrm{d}}(8 \mathrm{H})$ & --- \\
\hline$\left[\mathrm{HgL}^{3} \mathrm{Cl}_{2}\right]$ & $1.75^{\mathrm{s}}(4 \mathrm{H})$ & & $3.81^{\mathrm{s}}(12 \mathrm{H})$ & $2.50^{\mathrm{m}}(8 \mathrm{H})$ & $7.16^{\mathrm{d}}(8 \mathrm{H}) 7.52^{\mathrm{d}}(8 \mathrm{H})$ & \\
\hline
\end{tabular}

* May be mixed with solvent peak, $s=$ singlet, $d=$ doublet, $m=$ multiplet, $b=$ broad

Based on analyses, conductance, magnetic, electronic, infrared and proton magnetic resonance spectral studies, a distorted octahedral geometry involving four $\mathrm{N}$ atoms of tetraazamacrocycle and $2 \mathrm{Cl}$ may be proposed for these $\mathrm{d}^{10}$ metal complexes. 


\section{Acknowledgment}

The authors are thankful to Maharshi Dayanand University, Rohtak for providing the necessary facilities.

\section{References}

1. Jones P G, Carman M and Arellano R D, J Chem Soc., 1996, 2713.

2. Kienitz C O, Thone C and Jones P G Jones, Inorg Chem., 1996, 35(13), 3990-3997.

3. Nishibayashi Y, Segawa K, Singh J D, Fukuzawa S I, Ohe K and Uemura S, Organometallics, 1996, 15, 370-379.

4. Nishibayashi Y, Singh J D, Arikawa Y, Uemura S and Hidai M, J Organometal Chem., 1997, 531, 13.

5. Chang Y, Emge T J and Brennan J G, Inrog Chem., 1996, 35, 342, 7339.

6. Steigerwalk M L and Sprinkle C R, J Am Chem Soc., 1987, 109, 7200.

7. Hirpo W, Dhingra S, Sutorik A C and Kanatzidis M G, J Am Chem Soc., 1993, 115, 1597-99.

8. $\quad$ Gange R R, Allison J L, Gall S and Kovac C A, J Am Chem Soc., 1997, 99, 7170.

9. Martin J W L, Johnson J H and Curtis N F, J Chem Soc Dalton Trans., 1978, 68-76.

10. Hughes M N, Inorg Chem Biological Process; $2^{\text {nd }}$ Edn, Wiley, New York, 1981.

11. Casella L, Gullotii M, Gioia L D, Monzani E and Chillemi F, J Chem Soc., Dalton Trans., 1991, 2945.

12. James S R and Margerum D W, Inorg Chem., 1980, 19, 2784.

13. Levason W, Orchard S D and Reid G, Coord Chem Rev., 2002, 225, 159.

14. Levason W and Reid G, J Chem Soc Dalton Trans., 2001, 2953.

15. Singh A K and Sharma S, Coord Chem Rev., 2000, 209, 49.

16. Levason W and Reid G, Handbook of Chalcogen Chemistry; RSC, 2006, p. 81.

17. Panda A, Coord Chem Rev., 2009, 253, 1947-1965

18. Srivastava S and Kalam A, J Indian Chem. Soc., 2006, 83, 563-567.

19. Nitu and Verma K K, J Chem Pharm Res., 2010, 2(4), 793-800.

20. Nitu and Verma K K, Int J Chem Sci., 2011, 9(1), 229-238.

21. Khandelwal B L, Kumar K and Berry F J, Inorg Chim Acta, 1981, 47, 135-137.

22. Khandelwal B L, Kumar K and Raina K, Synth React Inorg Met Org Chem., 1981, 11(1), 65.

23. Bergman J, Tetrahedron, 1972, 28, 3323.

24. Vogel A I, A Text Book of Quantitative Inorganic Analysis; $3^{\text {rd }}$ Edn, Longman, London, 1989, p.266, 324.

25. Geary W J, Coord Chem Rev., 1971, 7, 81-122.

26. Sonmezn M, Levent A and Sekerci M, Synth React Inorg Met Org Chem., 2003, 33, 1747.

27. Mukhopadhyay R, Bhattacharjee S and Bhattacharya R, J Chem Soc.,1944, 2799.

28. Rana V B, Singh P, Singh D P and Teotia M P, Polyhedron, 1982, 1, 377-380.

29. House D A and Curtis N F, J Am Chem Soc., 1964, 86, 1331.

30. Shakir M, Varkey S P and Hameed P S, Polyhedron, 1994, 13, 1355.

31 Panda A K., Panda A, Sutar S, Mishra P, Pradhan S, Ghos S and Pany S, J Indian Chem. Soc., 2009, 86, 908.

32. Srivastava S and Kalam A, Synth React Inorg Met Org Chem., 2004, 34, 1529.

33. Kulkarni Y D and Srivastava S, Indian J Chem., 1985, 22, 710.

34. Nakamoto K, Infrared and Raman Spectra of Inorganic and Coordination Compounds; $5^{\text {th }}$ Edn, Part-B, John Wiley and Sons, New York, 1997, 15-20.

35. Raina K and Khandelwal B L, Indian J Chem., 1976, 14A, 63.

36. Academic Press Hand Book Series - Hand Book of Proton NMR Spectra and Data, Edited by Asani Research Centre, Vol. 1, p. 47.

37. Menon S C, Singh H B, Patel R P and Kulshreshtha S K, J Chem Soc Dalton Trans., 1996, 1203. 


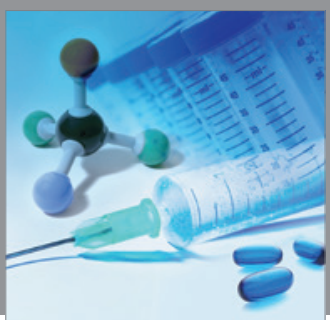

International Journal of

Medicinal Chemistry

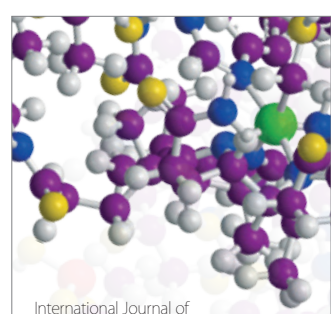

Carbohydrate Chemistry

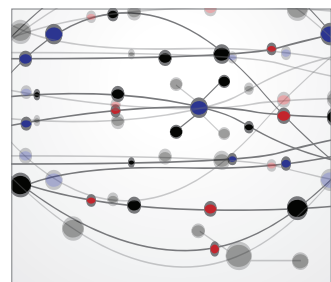

The Scientific World Journal
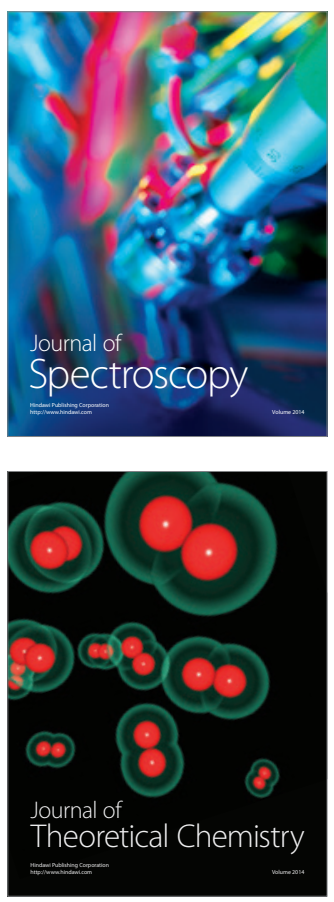
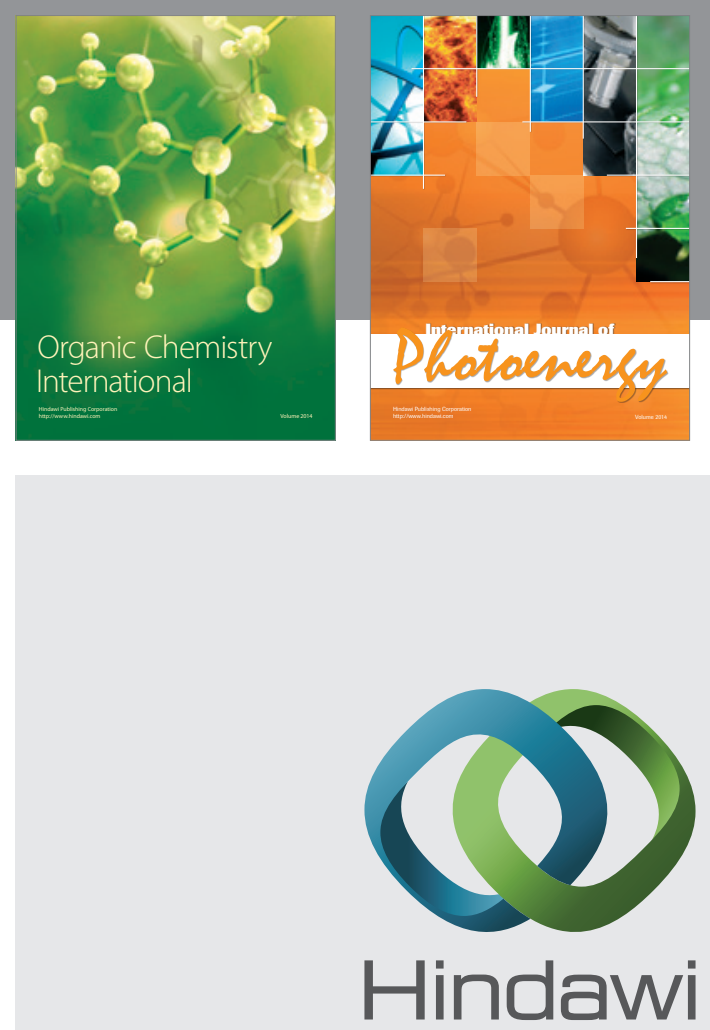

Submit your manuscripts at

http://www.hindawi.com
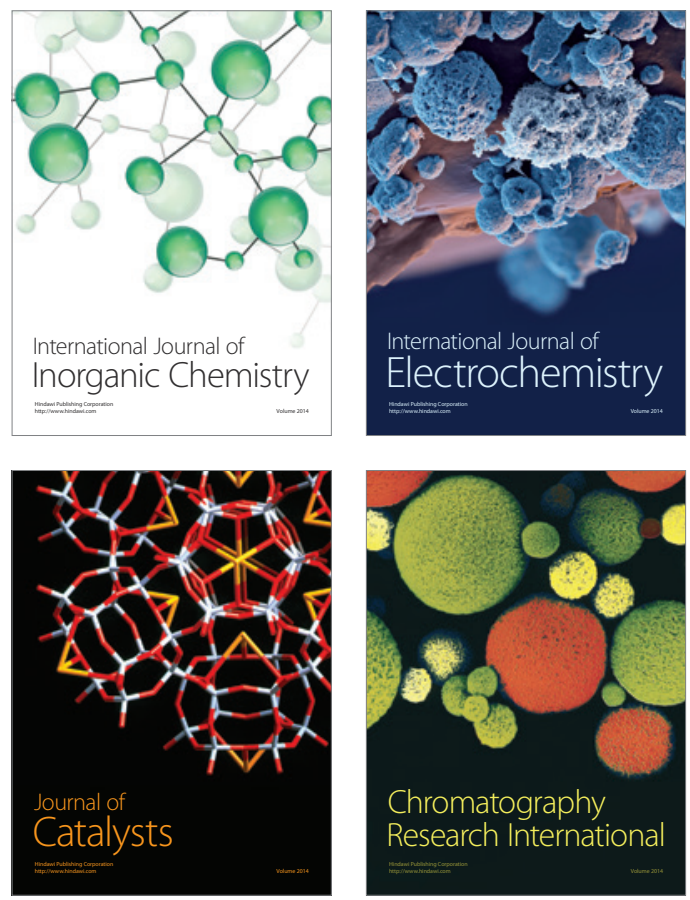
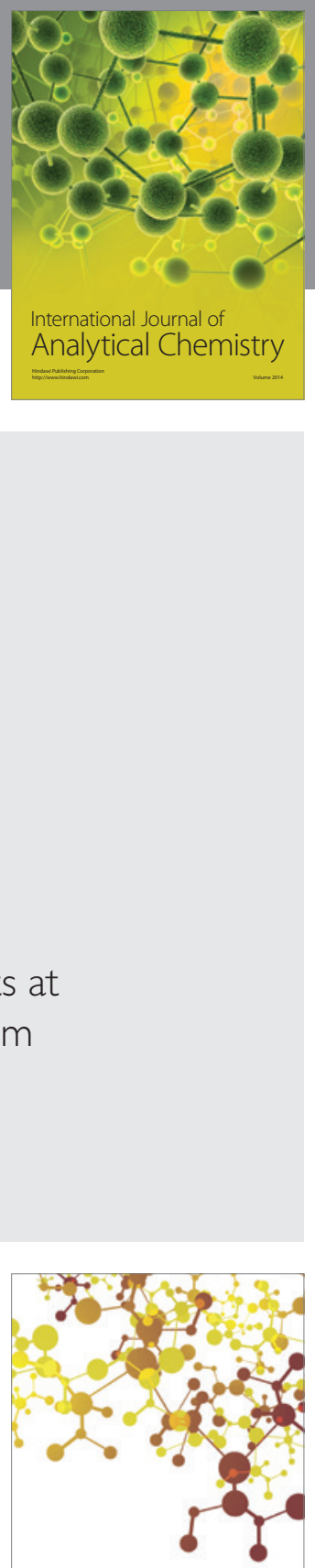

Journal of

Applied Chemistry
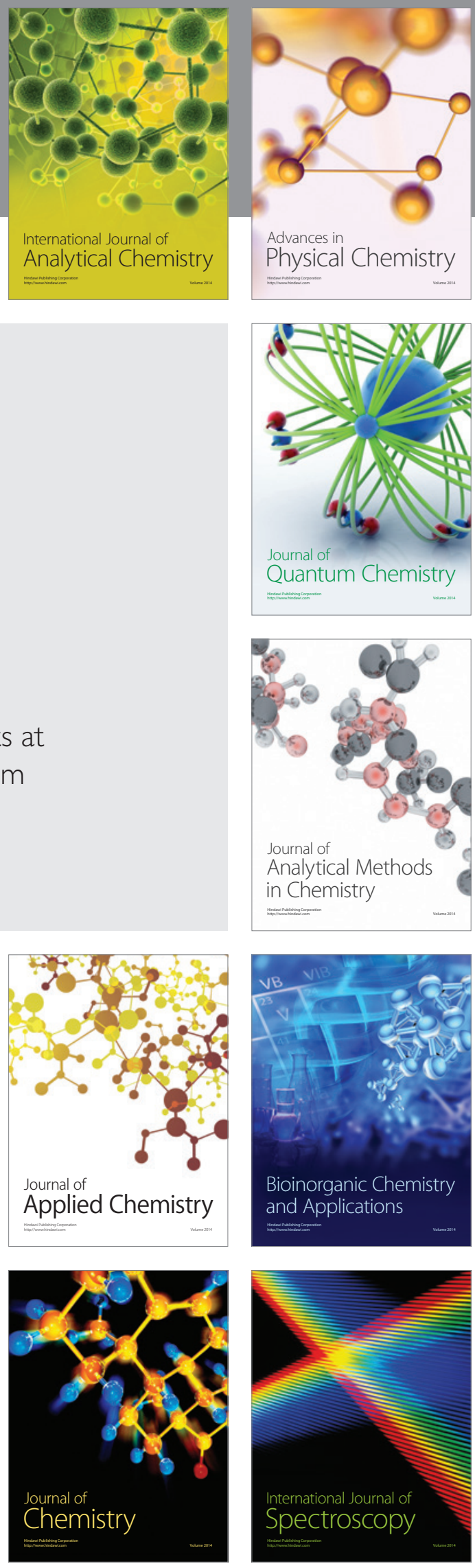